\title{
FORMATION OF P CYGNI LINE PROFILES IN RELATIVISTICALLY EXPANDING ATMOSPHERES. II. THE EFFECT OF THE CONTINUUM FREQUENCY SHAPE AND LIMB DARKENING OF THE CORE
}

\author{
DAMIEN HUTSEMÉKERS \\ Institut d'Astrophysique, 5 Av. de Cointe, B-4000 Liège, Belgium \\ Received 1993 February 25 ; accepted 1993 May 10
}

\begin{abstract}
In the framework of the relativistic Sobolev theory for the transfer of line radiation in spherically symmetric expanding atmospheres, we investigate the effect on P Cygni line profiles of the frequency shape of the underlying continuum (a power law), as well as the influence of an eventual limb darkening of the core.

We find that the effects due to limb darkening are small as in the classical limit. On the contrary, the line profiles strongly depend on the power-law index characterizing the continuum shape, even for moderate values of the terminal velocity of the flow. While the absorption part of the line profiles remains essentially unchanged, the emission part may be significantly displaced, enhanced, or lowered up to nearly complete disappearance. The equivalent width of the line profiles clearly depends on the power-law index.

The relevance of these results for interpreting the spectrum of broad absorption line (BAL) QSOs is briefly discussed. Considering values currently estimated for both the terminal velocity of the flow and the power-law index of the continnum, we find that the equivalent width of the emission-line profiles should be significantly smaller than the equivalent width of the absorption profiles, therefore possibly providing us with a natural explanation for the low emission/absorption-line ratios reported in the spectrum of some BAL QSOs.

Subject headings: line: formation - line: profiles - quasars: general — radiative transfer — relativity
\end{abstract}

\section{INTRODUCTION}

P Cygni line profiles characterized by relativistic Doppler velocities are currently observed in the spectrum of astronomical objects like supernovae and broad absorption line (BAL) QSOs (see, e.g., Weymann \& Foltz 1983).

In order to interpret more accurately these profiles, Hutsemékers \& Surdej (1990, hereafter Paper I) have generalized the Sobolev theory for the transfer of line radiation in expanding atmospheres to the case of special relativity. If the terminal velocity of the flow is sufficiently large $(\geq 0.2 c)$, the relativistic $P$ Cygni line profiles were found to significantly differ from those computed in the framework of the classical theory. Essentially, the emission part of the relativistic profiles appears sharper (on a frequency scale), mainly due to the redistribution of the scattered line photons in a frequency interval no longer symmetrical around the line center frequency, in accordance with the relativistic expression of the Doppler effect.

The aim of the present paper is to investigate the effect on relativistic P Cygni line profiles of the frequency dependence of the continuum (basically a power law) and an eventual limb darkening of the core. While the latter has no more influence than in the classical limit, the profiles were found to strongly depend on the continuum shape, with noticeable modifications of their equivalent widths.

In $\S 2$, we rewrite the expression of the line profile function derived in Paper I, accounting for the dependence in frequency and angle of the continuum intensity. Numerical examples are presented in $\S 3$, while discussion and conclusions form the last section.

\section{THE EXPRESSION OF THE LINE PROFILE}

As in Paper I, we consider a spherically symmetric atmosphere expanding around a central core of radius $r_{c}$. The flow is accelerated outward, $v(r)$ denoting the velocity of the flow at the distance $r$ from the core. The fluid is assumed populated by two-level atoms, the transition between the levels being characterized by the comoving line center frequency ${ }^{1} v_{0}$.

In the framework of the Sobolev approximation (Sobolev 1960), the source function as well as the total optical depth may be evaluated locally in each point of the envelope. Measured by an observer at rest, the line profile function at a frequency $v$, is therefore simply expressed by (see, e.g., Castor 1970; Rybicki \& Hummer 1978; Surdej 1979):

$$
E_{v} \propto \int_{\Sigma_{v}} S_{v}\left(1-e^{-\tau}\right) 2 p d p+\int_{\Sigma_{v}} I_{v}^{c} e^{-\tau} 2 p d p,
$$

where $S_{v}(r)$ is the Sobolev source function, $\tau(r, \mu)$ is the Sobolev optical depth given in Paper I (eq. [I.25]), $I_{v}^{c}$ is the intensity of the continuum emitted by the core, and $p$ is the impact parameter (cf. Fig. 1). The integration is performed over the so-called surfaces of equal frequency $v$, denoted by $\Sigma_{v}$, and defined by the relativistic Doppler relation $v=$ $v_{0} \gamma^{-1}(1-\mu \beta)^{-1}$, where $\beta=v(r) / c, \gamma$ is the Lorentz factor $1 / \sqrt{1-\beta^{2}}$ and $\mu=\cos \theta$ is the cosine angle between the direction of the photon (in this case the line of sight) and that of the flow. Examples of surfaces of equal frequency are shown in Paper I.

The source function evaluated at a given point in the envelope accounts for the intensity of radiation emitted by the core and able to penetrate that point. Its expression may be easily generalized from Paper I. Considering pure scattering of line photons, assuming complete redistribution in the comoving frame, and adopting for the intensity of the radiation

\footnotetext{
${ }^{1}$ Throughout this paper, we omit the subscript " 12 " used in Paper I, while the subscript " 0 " still indicates a quantity measured in the comoving frame. Also, equations referred to with a number preceded by " I" are equations given in Paper I.
} 


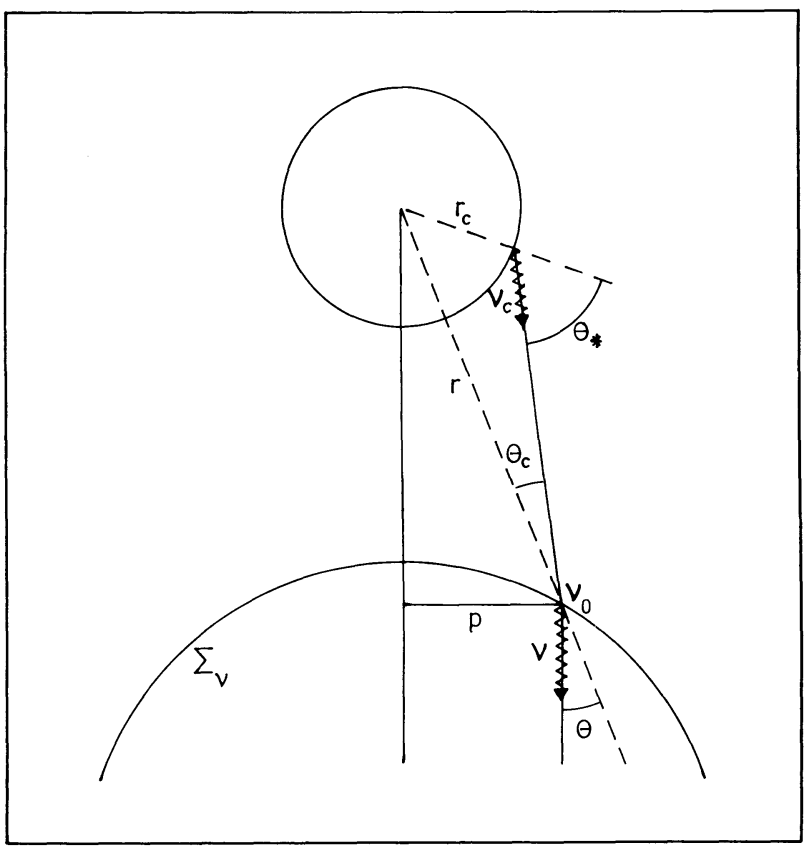

FIG. 1.-Schematic representation of the path of a photon emitted by the core at frequency $v_{c}$, interacting with the outflowing material at the line center frequency $v_{0}$ on the surface of equal frequency $\Sigma_{v}$, and finally escaping the flow toward the observer with the frequency $v$. To the angles $\theta, \theta_{c}$, and $\theta_{*}$ defined in this figure correspond the cosine angles $\mu, \mu_{c}$, and $\mu_{*}$.

emitted by the core in the continuum the form

$$
I_{v}^{c}\left(\mu_{*}\right)=I_{c}\left(\frac{v}{v_{0}}\right)^{\alpha} \Psi\left(\mu_{*}\right),
$$

where $\alpha$ is the power-law index and $\Psi\left(\mu_{*}\right)$ the limb-darkening law (cf. Fig. 1), the Sobolev source function evaluated in the comoving frame may be written

$$
S_{v_{0}}^{0}=\frac{\beta^{3}}{\beta^{1}} I_{c}
$$

with the penetration probability $\beta^{3}$ being expressed by

$$
\beta^{3}=\int_{1-2 W}^{1}\left(\frac{v_{c}}{v_{0}}\right)^{\alpha} \Psi\left(\mu_{*}\right) \eta_{c} \frac{1-e^{-\tau}}{\tau} \frac{d \mu_{c}}{2} .
$$

The escape probability $\beta^{1}$ has the same expression as in Paper I (eq. [I.22]). In equation (4), $v_{c}$ represents the observer-frame frequency of a photon emitted by the core in the continuum and able to interact at the point in the envelope where the source function is evaluated. In accordance with the Doppler relation, $v_{c}=v_{0} \gamma^{-1}\left(1-\mu_{c} \beta\right)^{-1}$. The factor $\eta_{c}=v_{0} / v_{c}=$ $\gamma\left(1-\mu_{c} \beta\right)$ accounts for the fact that we have no longer divided the intensity by $h v_{c}$ as done in Paper I. $W$ denotes the geometrical dilution factor $\left[1-\sqrt{1-\left(r_{c} / r\right)^{2}}\right] / 2$. It should also be pointed out that when computing $\beta^{3}, \tau(r, \mu)$ must be evaluated for $\mu=\mu_{c}$. Finally, let us notice that in deriving equation (4), we have implicitly assumed that the intensity of the continuum radiation is constant over the atomic absorption profile.

Using the transformation laws between the rest and comoving frames ([I.5]-[I.7]), the source function finally writes in the observer reference frame:

$$
S_{v}=\eta^{-3} \frac{\beta^{3}}{\beta^{1}} I_{c}
$$

where

$$
\beta^{3}=\int_{1-2 W}^{1} \Psi\left(\mu_{*}\right) \eta_{c}^{1-\alpha} \frac{1-e^{-\tau}}{\tau} \frac{d \mu_{c}}{2}
$$

and

$$
\beta^{1}=\int_{-1}^{+1} \frac{1-e^{-\tau}}{\tau} \frac{d \mu}{2 \eta^{2}} .
$$

In these relations, $\eta=v_{0} / v=\gamma(1-\mu \beta)$.

Due to the fact that in equation (1), the intensity is integrated over the surfaces of equal frequency, the variables $r, p$, and $v$ are not independent, and the line profile function may be conveniently evaluated using the dimensionless frequencies $x$ and $x^{\prime}$, which also provide us with a length scale physically more adequate for the numerical integration. The dimensionless frequency $x$ is simply defined by

$$
x=-\frac{v-v_{0}}{v_{\max }-v_{0}},
$$

where $v_{\max }$ represents the largest observable frequency in the profile, corresponding to the outflow terminal velocity $v_{\infty}=$ $c \beta_{\infty}$. With the help of the expressions given in Paper I for $x$ (eq. [I.12]) and $x^{\prime}=x(\mu=+1)$, we easily find that $2 p d p=-4 r^{2} \eta^{2}\left(\tau^{\prime} / \tau\right)\left(x^{R}-x^{\prime}\right)^{-1} d x^{\prime}, \quad \tau^{\prime}=\tau(r, \mu=+1) \quad$ representing the Sobolev optical depth evaluated along a radial direction, and $x^{R}=-x^{\prime} \sqrt{(1-\beta) /(1+\beta)}$ (cf. eqs. [I.26] and [I.35]).

Denoting by $E_{v}^{C}$ the continuum flux which reaches the observer in the absence of the expanding atmosphere, i.e. $E_{v}^{C} \propto$ $r_{c}^{2} I_{c} \bar{\Psi}\left(v / v_{0}\right)^{\alpha}$ where

$$
\bar{\Psi}=2 \int_{0}^{1} \Psi\left(\mu_{*}\right) \mu_{*} d \mu_{*},
$$

it is now straightforward to write the final expression of the normalized line profile function:

$$
\frac{E}{E^{C}}(x)=\frac{E^{S}}{E^{C}}(x)+\frac{E^{A}}{E^{C}}(x),
$$

where

$$
\frac{E^{S}}{E^{c}}(x)=\bar{\Psi}^{-1} \eta^{\alpha-1} \int \frac{\beta^{3}}{\beta^{1}} \frac{1-e^{-\tau}}{\tau} \frac{4 \tau^{\prime}\left(r / r_{c}\right)^{2}}{x^{R}-x^{\prime}} d x^{\prime},
$$

and

$$
\begin{array}{ll}
\frac{E^{A}}{E^{C}}(x)=2 \bar{\Psi}^{-1} \int_{0}^{1} \Psi\left(\mu_{*}\right) e^{-\tau} \mu_{*} d \mu_{*} & \text { if } x \leq x_{*} \\
\frac{E^{A}}{E^{C}}(x)=1 & \text { if } x \geq x_{*} .
\end{array}
$$

The quantity $x_{*}$ and the limits of integration in equation (11) may be evaluated exactly as in Paper I (cf. eqs. [I.45]-[I.48]). In this relation, $\eta=v_{0} / v=\left(1-x B_{\infty}\right)^{-1}$ where $B_{\infty}=$ $\sqrt{\left(1+\beta_{\infty}\right) /\left(1-\beta_{\infty}\right)}-1$ (cf. eq. [8]).

These relations generalize the relativistic expression of the line profile function established in Paper I, accounting for a power-law frequency dependence of the continuum and an eventual limb darkening of the core. The main difference resides in the evaluation of the source function. We easily recover the expressions of Paper I when $\Psi\left(\mu_{*}\right)=1$ and $\alpha=1$, i.e., in the absence of any limb darkening and assuming con- 
stant the number of photons $\left(I_{v}^{c} / h v\right)$ emitted by the core in the continuum. With $\alpha=1$, the source function takes its simplest form, readily derivable in terms of a probabilistic approach which manipulates photon numbers rather than intensities. This approach was that followed in Paper I. When $\alpha$ is not equal to 1 , the additional factor $\left(\eta_{c} / \eta\right)^{1-\alpha}=\left(v / v_{c}\right)^{1-\alpha}$ which appears in combining equations (6) and (11), simply accounts for the fact that the number of photons in the continuum at the absorption frequency $v_{c}$ is not equal to the number of photons in the continuum at the reemission frequency $v$.

Let us finally notice that assuming the continuum flux constant over the wavelength interval which corresponds to the line profile (an approximation frequently used when computing profiles) results in line profiles different from those computed in assuming that the continuum flux is constant over a frequency interval, simply because the former approximation corresponds to a power-law shaped continuum with $\alpha=-2$.

\section{NUMERICAL APPLICATIONS}

By comparing equations (6), (11), and (12) to their equivalent (I.30), (I.45), and (I.46) we can see that the differences may be readily accounted for in the numerical code used in Paper I. We therefore consider the same parameterization as well as the same types of velocity and opacity distributions.

We have first investigated the influence of the limbdarkening law on the line profiles. An example is given in Figure 2. We found that this influence is very similar to what has been previously reported from computations in the classical limit: the effect remains small, affecting mainly the line center (see, e.g., Surdej 1981, and references therein). This result is not surprizing since the influence of the limb darkening is actually important in the regions of the envelope near the core, i.e., in regions where the velocities are generally not yet relativistic.

Much more important and interesting is the dependence of the line profiles on the shape of the underlying continuum.

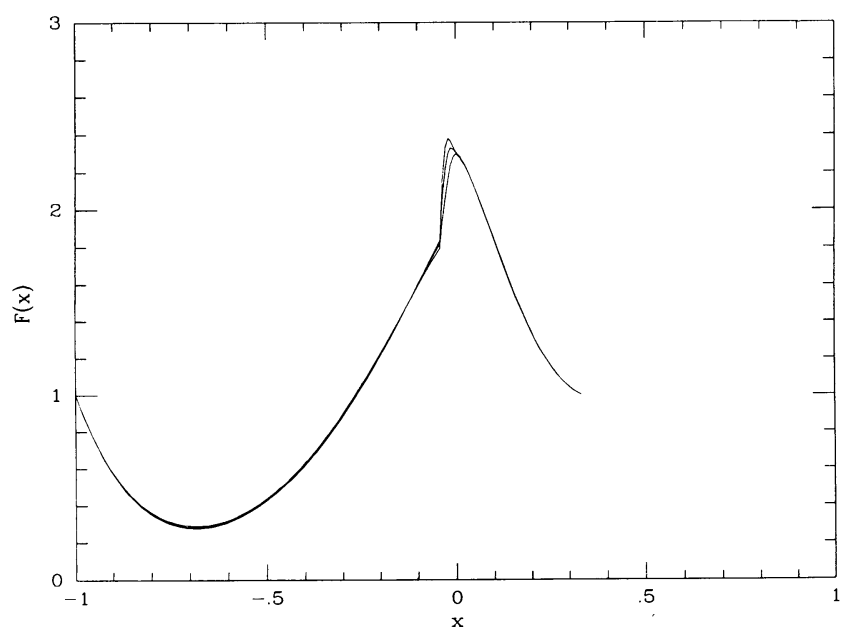

FIG. 2.-Example of relativistic P Cygni line profiles computed with limb darkening of the core: $\Psi\left(\mu_{*}\right)=\mu_{*}^{*}$, where $n=0,1$, and 2 . The higher $n$, the higher the emission line intensity. $F(x)=E / E^{c}$ and $x$ is the dimensionless frequency (cf. eqs. [8], [10]). The power-law index $\alpha$ is equal to 1 . The terminal velocity $\beta_{\infty}=0.8$. The opacity and velocity distribution characterizing the expanding envelope were parameterized as in Paper I (cf. eq. [1.70]). The adopted velocity law is $\beta(r)=\beta_{c}+\left(\beta_{\infty}-\beta_{c}\right)\left(1-r_{c} / r\right)$ with $\beta_{c} / \beta_{\infty}=0.1$. For all the profiles, the parameter characterizing the optical depth, $W_{1}^{R, 0}$, was set to $W_{1}^{R, 0}=1$. The adopted opacity distribution is $\tau^{r}(\beta)=1-\beta / \beta_{\infty}$
Figure 3 illustrates $\mathrm{P}$ Cygni type profiles computed for different values of the power-law index $\alpha$. Strong differences between the profiles (and their equivalent widths) may be noticed, the emission being clearly enhanced when $\alpha$ increases. For some line profiles, the position of the maximum of the emission significantly changes, while, in other cases, the emission may strongly decrease up to nearly complete disappearance. The amplitude of these effects depends on $\beta_{\infty}$ in the sense that it is greater for larger values of $\beta_{\infty}$. When $\beta_{\infty} \rightarrow 0$, i.e., in the classical limit, the profiles are essentially independent on $\alpha$, provided that reasonable values of this latter quantity are still considered.

This behavior may be easily understood. Photons absorbed at a dimensionless frequency $x \leq 0$ are reemitted at larger dimensionless frequencies, with conservation of their number. When $\beta_{\infty}$ is large enough, the line profile extends over a frequency range on which the continuum level may change significantly due to its $v^{\alpha}$ dependence. Depending on the value of $\alpha$, the level of the continuum which underlies the emission may be smaller or greater than its level at the absorption frequency $x$, such that, when normalized to the continuum, the emission part of the profile may be enhanced or lowered. In the case of shell-type profiles, the maximum of the emission may be displaced because the emission part of these profiles is more or less flat-topped so that the shape of the emission profile basically reflects, after normalization, the frequency dependence of the continuum. If the core emits the same number of photons at all frequencies, i.e., when $\alpha=1$, the equivalent width of the emission part of the profile is expected to be nearly equal to that of the absorption part, the expanding atmosphere being assumed spherically symmetric. ${ }^{2}$ However, for values of $\alpha$ not equal to 1 , the equivalent width of the emission may strongly differ from that of the absorption. Strictly speaking, the effects described above are not purely relativistic, but, for reasonable values of $\alpha$, they may only appear whether large expansion velocities prevail in the envelope, therefore necessitating the use of the relativistic expression for the line profile function.

Let us finally notice that when a wavelength scale is considered rather than a frequency scale, the shape of the profiles is different, the red part of the emission being more extended than the blue part, due to the asymmetry of the relativistic Doppler effect (cf. the discussion in Paper I). However, whatever the scale, the dependence of the profiles on the continuum shape is qualitatively identical.

\section{DISCUSSION AND CONCLUSIONS}

Considering a power-law frequency shape for the intensity of the radiation emitted in the continuum, as well as an eventual limb darkening of the core, we have generalized the expression of $\mathbf{P}$ Cygni line profiles formed in relativistically expanding atmospheres.

On the basis of numerical simulations, we found that the effect on the profiles of the limb darkening of the core remains small, as in the classical limit. On the contrary, the P Cygni line profiles strongly depend on the power-law index $\alpha$ characterizing the shape of the underlying continuum. Depending on $\alpha$, the emission part of the profile may be displaced, enhanced, or lowered up to nearly complete disappearance.

\footnotetext{
${ }^{2}$ In fact, the equivalent widths of the emission and absorption profiles are slightly different because the part of the expanding atmosphere occulted by the core cannot contribute to the emission profile. The importance of this occulted part is nevertheless small for the models considered here.
} 

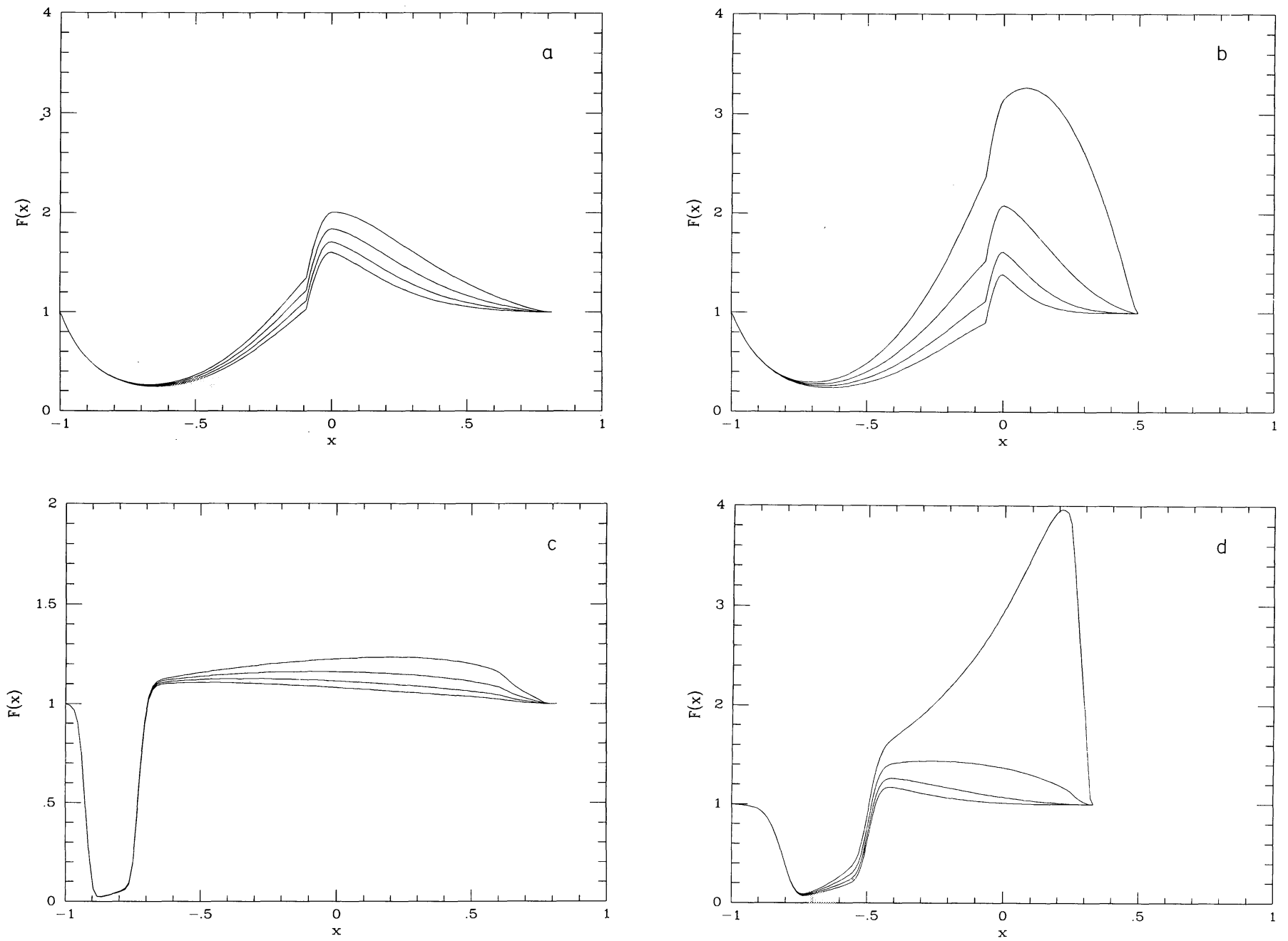

FIG. 3.-Examples of relativistic P Cygni line profiles computed with no limb darkening of the core $\left[\Psi\left(\mu_{*}\right)=1\right]$ and adopting a power-law shape for the underlying continuum. The four profiles illustrated in each figure were calculated for different values of the power-law index: $\alpha=-3,-1,1,3$. The profiles computed with the largest value of $\alpha$ are those with the most intense emission. $F(x)=E / E^{C}$ and $x$ is the dimensionless frequency (cf. eqs. [8], [10]). The terminal velocity $\beta_{\infty}$ is equal to $0.2(a, c), 0.6(b)$ and $0.8(d)$. The opacity and velocity distributions characterizing the expanding envelope were parameterized as in Paper I (cf. eq. [I.70]). The adopted velocity law is $\beta(r)=\beta_{c}+\left(\beta_{\infty}-\beta_{c}\right)\left(1-r_{c} / r\right)$ with $\beta_{c} / \beta_{\infty}=0.1$. For all the profiles, the parameter characterizing the optical depth, $W_{1}^{R, 0}$, was set to $W_{1}^{R, 0}=1$. The adopted opacity distributions are $\tau^{r}(\beta)=1-\beta / \beta_{\infty}(a, b)$ and $\tau^{r}(\beta)=\exp \left[-\left(\beta-\beta_{L}\right)^{2} / \beta_{\sigma}^{2}\right]$ where $\beta_{L} / \beta_{\infty}=0.85$ and $\beta_{\sigma} / \beta_{\infty}=0.05(c, d)$. The illustrated profiles are typical of an expanding atmosphere with a continuous outflow $(a, b)$ and an opacity shell $(c, d)$.

Since some of these effects are already significant for moderate values of $\beta_{\infty}$, currently observed in the spectrum of BAL QSOs or supernovae, they should be taken into account when interpreting the line profiles or simply when measuring redshifts from the emission lines.

The case of BAL QSOs is especially interesting: while their spectra show strong absorption troughs, the emission lines are often weaker. Considering that resonance scattering in spherically symmetric envelopes should produce emission equivalent in strength to the absorption, this fact has been interpreted as evidence for a small covering factor of the absorbing material (Turnshek et al. 1980; Turnshek 1984; see also the discussion by Surdej \& Hutsemékers 1987). However, if we adopt the typical $v^{-1}$ shape characteristic of QSO continua, we naturally expect from our calculations smaller equivalent widths for the emission than for the absorption. For example, the equivalent width of the emission part of the profile illustrated in Figure $3 a$ and computed with $\alpha=-1$ and $\beta_{\infty}=0.2$, is already about a factor 2 smaller than the equivalent width of the absorption. Stronger effects are expected if the law characterizing the continuum shape is steeper $(\alpha \leq-2$ has been reported for the continuum of some BAL QSOs by Junkkarinen, Burbidge, \& Smith, 1987). Also, the presence of black, detached absorption-line profiles with little or no emission, has sometimes been reported in the spectrum of BAL QSOs and presented as an argument against line formation by resonance scattering in a symmetric envelope. As shown in Figure 3, such profiles are not too difficult to reproduce with shell-type opacity enhancements in the envelope and $\alpha$ sufficiently small to mask most of the emission.

Among the BAL QSOs, Weymann et al. (1991) recently identified a subclass of objects which display in their spectra strong absorption troughs in low ionization species in addition to the usual high-ionization broad absorption lines. The shape of the continuum characterizing these "low-ionization" BAL QSOs is significantly different from that characterizing the other "high-ionization" BAL QSOs. Sprayberry \& Foltz (1992) interpreted this difference as due to the presence of dust around 
the low-ionization BAL QSOs, and, after correcting for the dust extinction, they found that the composite spectra of both classes of BAL QSOs may be reconciled, except the strength of Iol the emission lines which remains smaller in the spectrum of the 2-1 low-ionization BAL QSOs. If we accept to interpret, at least partially, these profiles in the framework of a P Cygni type model, the emission line differences may be the consequence of the continuum shape difference observed between these two classes of BAL QSOs. In this case, the dust containing region should be located inside of the BAL region.
While the effects discussed in the present paper can certainly not explain all the peculiarities of the line profiles observed in the spectrum of BAL QSOs, it is clear that they sufficiently affect the profiles to be taken into account when interpreting, even qualitatively, the observations.

It is a pleasure to thank Jean Surdej for a careful reading of the manuscript. This research is supported in part by contract ARC 90/94-140 "Action de recherche concertée de la Communauté Française" (Belgium).
Castor, J. I. 1970, MNRAS, 149, 111

Hutsemékers, D., \& Surdej, J. 1990, ApJ, 361, 367 (Paper I)

Junkkarinen, V. T., Burbidge, E. M., \& Smith, H. E. 1987, ApJ, 317, 460

Rybicki, G. B., \& Hummer, D. G. 1978, ApJ, 219, 654

Sobolev, V. V. 1960, Moving Envelopes of Stars (Cambridge: Harvard Univ. Press)

Sprayberry, D., \& Foltz, C. B. 1992, ApJ, 390, 39

Surdej, J. 1979, A\&A, 73, 1

Surdej, J.1981, Ap\&SS, 79, 213

\section{REFERENCES}

Surdej, J., \& Hutsemékers, D. 1987, A\&A, 177, 42

Turnshek, D. A. 1984, ApJ, 278, L87

Turnshek, D. A., Weymann, R. J., Liebert, J. W., Williams, R. E., \& Strittmatter, P. A. 1980, ApJ, 238, 488

Weymann, R. J., \& Foltz, C. 1983, in Proc. 24th Liège Internat. Astrophys. Colloq., Quasars and Gravitational Lenses, ed. J. P. Swings (Liège: Univ. Liège), 538

Weymann, R. J., Morris, S. L., Foltz, C. B., \& Hewett, P. C. 1991, ApJ, 373, 23 